\title{
Analysis, design, and management of health care systems
}

\author{
Andrea Matta ${ }^{1}$ Alain Guinet ${ }^{2} \cdot J_{\text {Jingshan } \mathrm{Li}^{3} \cdot \operatorname{Evren~Sahin}}^{4}$. \\ Nico Vandaele ${ }^{5}$
}

The way in which health care service organizations are managed affects their global system performance and needs to be re-designed in order to address the requirements of the new environment. The primary objective of this Special Issue of the Flexible Services and Manufacturing (FSM) Journal is to reflect recent developments to improve the service delivery in health care systems. A total of 44 submissions were received. After multiple rounds of rigorous peer reviewing processes, 14 of them have been accepted. These papers cover care delivery management from ambulatory service to hospital care with new theoretical

Andrea Matta

andrea.matta@polimi.it

Alain Guinet

alain.guinet@insa-lyon.fr

Jingshan Li

jingshan@engr.wisc.edu

Evren Sahin

evren.sahin@ecp.fr

Nico Vandaele

Nico.Vandaele@kuleuven.be

1 Department of Mechanical Engineering, Politecnico di Milano, Via La Masa 1, 20156 Milano, Italy

2 Département Génie Industriel, INSA de Lyon, 19 avenue Jean Capelle, 69621 Villeurbanne cedex, France

3 Department of Industrial and Systems Engineering, University of Wisconsin-Madison, 1513 University Avenue, Madison, WI, USA

4 Laboratoire Génie Industriel, Centrale Supélec, Grande Voie des Vignes, 92290 Châtenay-Malabry, France

5 Research Center of Operations Management, KU Leuven, E. Sabbelaan 53, 8500 Kortrijk, Belgium 
contributions and real world examples illustrating the state-of-the-art research in this field. It is characterized by interdisciplinarity and a symbiose between theory and application. Additionally, a mix of technical, economical and human values seems to be inherent to the field of health care system engineering. Specifically, the following parts are included:

- Primary, specialty, and home care

This part addresses staffing and scheduling issues in primary care,specialty clinics and home care.

Zhong, Lee, Williams, Kraft, Sleeth, Welnick, Hauschild and Li investigate the optimal staffing composition of physicians (MD) and medical assistants (MA) in primary care clinics. Both Markov chain and discrete event simulation models are developed to examine the effects of workload shifting and to identify the proper ratio of MDs to MAs. The results articulate that the optimal staffing ratio is achieved when the workloads of MDs and MAs are balanced. Such optimality is not affected by system variations in terms of patient volume, neither by the arrival and service time distributions.

Borgman, Vliegen, Boucherie and Hans study unscheduled arrivals and reprioritization in a radiology department for CT diagnostic tests. A discrete event simulation model is developed to evaluate the performance of a given appointment schedule, and a local search heuristic is used to optimize the schedule. The results suggest a considerable decrease of waiting time for scheduled patients, while still treating unscheduled patients on time.

Bendavid, Marmor and Shnits introduce an optimal appointment scheduling system under rigid standby time and pre-determined quality of service in the cancer diagnostic process. An equivalent deterministic problem based on simulation and mixed-integer linear programming is formulated to solve the stochastic problem. It is found that the proposed policy can achieve stable and fair quality of service for patients and the policy performs better than the benchmark policy.

Fikar and Hirsch study a car and trip sharing concept to delivery and pick up nurses for home care services. An event-driven biased-randomized heuristic, a postoptimization procedure, and metaheuristics are used for numerical evaluation. It is shown that trip sharing performs best if long services and long delays exist, and if both geographical areas and patient clusters are randomly distributed.

- Emergency care and laboratory service

High performing emergency services are critical for patient safety and health care quality. This part studies emergency care admission and ambulance assignment, as well as laboratory scheduling.

Lee and Lee introduce an admission control study in an emergency department in the aftermath of a mass casualty incident. A finite horizon Markov decision process (MDP) model is formulated to determine patient admission decisions. Timedependent patient arrivals and reward functions are considered and structural properties are reviewed. The performance and advantage of optimal policies obtained from the MDP model are demonstrated through virtual experiments. 
Boujemaa, Jebali, Hammami, Ruiz and Bouchriha intend to design a two-tiered emergency medical service (EMS) system with out-of-hospital acute care and transportation. A two-stage stochastic programming location-allocation model is proposed to simultaneously determine the location of ambulance stations and serving areas, as well as the number and type of ambulances. A sampling average approximation algorithm is used to solve the problem efficiently.

Sung and Lee present a scenario-based ambulance location model to evaluate the ambulance availability under a dispatching policy. Two-stage stochastic programming is used to represent the temporal variations in call arrivals and constraints are embedded to ensure that the ambulance assignment process follows a given dispatching policy. Then a logic-based Benders decomposition algorithm is proposed to solve the problem.

Leeftink, Boucherie, Hans, Verdaasdonk, Vliegen and van Diest study a batch scheduling issue present in histopathology laboratories. Modeling tissue processors as batch processing machines, a 2-phased decomposition approach is introduced to solve the problem and to improve the spread of workload as well as to reduce the tardiness. Using historical data, it shows that the decomposition method could significantly reduce peaks in workload and turnaround times. Such an approach is currently being implemented in a large university medical center in The Netherlands.

\section{- Surgical care}

Surgery is one of the most important care delivery processes in the hospital, where operating room scheduling has attracted substantial attention.

Zeng, Xie, Menaker, Sanford-Ring and Li introduce an analytical model to quickly and accurately evaluate the expected idle time and waiting time of operating room schedules in an orthopedic surgery department. Closed formulas are derived for the two-surgery case. Then an iterative procedure is presented for multi-surgery scenarios, where every two surgeries are aggregated into one using the two-surgery formula. This is continued to the next one, until all surgeries are aggregated into one. The method is validated by historical data and a software tool is described as well.

Molina-Pariente, Hans and Framinan propose a Monte Carlo optimization method to study the stochastic operating room scheduling problem. The assignment of the intervention date and surgery room on the waiting list, as well as the minimization of the under/overtime costs and the cost of exceeding capacity constraints, are considered. The sample average approximation method is used to combine an iterative greedy local search method and Monte Carlo simulation.

A mixed integer multi-objective model is presented by Cappanera, Visintin and Banditori to determine the number and typology of surgeries in operating rooms. The objectives are to optimize patient due date fulfilment rate, operating room and bed utilizations, and the number of scheduled surgeries, which all reflect the heterogeneous priorities of the different stakeholders. Based on data from a children's hospital, a goal programming approach is used to solve the multiobjective problem. 
Alfieri, Listorti and Matta analyze the relationship between a higher volume of activity and better results in order to help the allocation of surgery interventions among health care structures. Different objective functions are studied to drive the allocation process towards better health conditions for patients. A decision support tool is provided for health policy makers, and three case studies are carried out to test the approach. The results are also compared to the data from the Italian National Outcome Evaluation Program.

- Hospital wide analysis

The last part focuses on analysis of the hospital wide performance.

Gross, Fugener and Brunner develop a mixed-integer linear programming model to create updated duty and workstation rosters simultaneously following absences of scheduled personnel. A case study is applied to schedule a hospital with 133 physicians, 17 duties, and 20 workstations in a 4 weeks planning horizon. It is demonstrated that the model can achieve near-optimal results with reasonable computational efforts, which can be used to manage trade-off between quality goals and plan stability.

Mahulea, Soriano and Colom introduce a Petri-net based modular approach for modeling healthcare systems. Two phases are included in each module: the sequences of treatments and cares, and the resources necessary to perform them. By fusing the inputs and outputs of the modules and adding patient information, a global model is obtained, which has equivalent behavior to a well-known class of resource allocation systems. Finally, a case study is described to illustrate the results.

In summary, analysis, design and management of a health care system is of significant importance for health care organizations to improve their performance, efficiency, quality and safety. Developing analytical and practical solutions is the key for successful care delivery. We hope this special issue can serve to intrigue more in-depth studies to achieve this goal.

Andrea Matta is Associate Professor at the Department of Mechanical Engineering of Politecnico di Milano. He graduated in Industrial Engineering at Politecnico di Milano where he develops his teaching and research activities since 1998. Distinguished Professor at the School of Mechanical Engineering of Shanghai Jiao Tong University from 2014 to 2016. He has been visiting professor at Ecole Centrale Paris (France), University of California at Berkeley (USA), and Tongji University (China). He is Guest Professor at the School of Mechanical Engineering of Shanghai Jiao Tong University. He is scientific responsible of the Research Area Design and Management of Manufacturing Systems at MUSP (Laboratory for Machine Tools and Production Systems). His research area includes analysis, design, and management of manufacturing and health care systems. He is Editor in Chief of Flexible Services and Manufacturing Journal, editorial board member of OR Spectrum journal and IEEE Robotics and Automation Letters journal. He was awarded the Shanghai One Thousand Talent and Eastern Scholar.

Alain Guinet is full University Professor in the Industrial Engineering Department of INSA (Institut National des Sciences Appliquées) de Lyon (France). He received a Ph.D. in 1983 and an Accreditation to Supervise Research (Habilitation à Diriger les Recherches) in 1992. He teaches: Operation Research, Staffing and Scheduling, Business Process Reengineering. His research activity is based on hospital management problems such as operating theatre control, hospital regrouping management, emergency 
network reengineering, home care resource coordination, hospital supply chain, and logistics for disaster management. His scientific investigations include: human and material resources dimensioning, planning and scheduling, production network reengineering, defense, logistics.

Jingshan Li received the Ph.D. degree in electrical engineering-systems from University of Michigan, Ann Arbor, in 2000. He was a Staff Research Engineer at the Manufacturing Systems Research Laboratory, General Motors Research and Development Center, Warren, MI from 2000 to 2006, and was with Department of Electrical and Computer Engineering and Center for Manufacturing, University of Kentucky, Lexington, KY from 2006 to 2010. He is now a Professor in Department of Industrial and Systems Engineering, University of Wisconsin, Madison, WI. He received 2010 NSF Career Award, 2009 IIE Transactions Best Application Paper Award, 2005 IEEE Transactions on Automation Science and Engineering Best Paper Award, 2006 IEEE Early Industry/ Government Career Award in Robotics and Automation, and multiple awards in international conferences. He is an IEEE Fellow, a Senior Editor of IEEE Transactions on Automation Science and Engineering and IEEE Robotics and Automation Letters, Department Editor of IIE Transactions and Associate Editor of International Journal of Production Research, Flexible Service and Manufacturing Journal, and International Journal of Automation Technology, and was an Associate Editor of Mathematical Problems in Engineering. His primary research interests are in modeling, analysis, and control of manufacturing and healthcare systems.

Evren Sahin is Professor at CentraleSupelec where she teaches Operations Management and Supply Chain Management. She received her Master degree (2001) and her Ph.D. (2004) in Industrial Engineering from Ecole Centrale Paris. Her research area includes the design and analysis of production and distribution systems for products and services. She is interested in optimizing service processes, with a particular focus on health care. She has been involved in several national projects with hospitals, emergency medical services and home care services. She has published more than 50 papers in international journals and conference proceedings. She has been visiting researcher at MIT in 2008.

Nico Vandaele holds a degree Commercial Engineering (1990) and obtained a Ph.D. in Applied Economics, Operations Research and Operations Management from KU Leuven in 1996. He is currently Full Professor Operations Management at the KU Leuven, Faculty of Business and Economics. He is holder of the GSK Research Chair on Access-to-Medicines. He is a research member of the Research Center of Operations Management. He is also a visiting researcher at CORE and IAG (Université Catholique de Louvain). He teaches courses in operations research, operations management and supply chain management. His research interests are situated in supply chain design, modeling of manufacturing and service systems, performance measurement, the design of planning systems, sales and operations management, factory physics, health care management and traffic modeling. He is co-founder Xathlon, a KU Leuven spin-off company and he was executive director of the Innovation and Incubation Center at Kortrijk. 